\title{
Boundary Sentinels with Given Sensitivity
}

\author{
Gisèle Massengo Mophou and Jean-Pierre Puel
}

\author{
Département de Mathématiques et Informatique \\ Université des Antilles et de La Guyane \\ Campus Fouillole \\ 97159 Pointe-à-Pitre — Guadeloupe (FWI) \\ Gisele.Mophou@univ-ag.fr
}

\author{
Laboratoire de Mathématiques de Versailles \\ Université de Versailles St Quentin \\ 45 avenue des Etats Unis \\ 78035 Versailles - France \\ jppuel@math.uvsq.fr
}

\begin{abstract}
The notion of sentinels with given sensitivity was introduced by J.-L. Lions in [10] in order to identify parameters in a problem of pollution ruled by a semilinear parabolic equation. He proves that the existence of such sentinels is reduced to the solution of exact controllability problem with constraints on the state. Reconsidering this notion of sentinels in a more general framework, we prove the existence of the new sentinels by solving a boundary null-controllability problem with constraint on the control. Our results use a Carleman inequality which is adapted to the constraint.
\end{abstract}

Key words: heat equation, optimal control, controllability, Carleman inequalities, sentinels.

2000 Mathematics Subject Classification: 35K05, 35K15, 35K20, 49J20, $93 \mathrm{~B} 05$.

\section{Introduction}

In the models of boundary pollution problem, initial data are not completely known. Boundary conditions also are either unknown or known only on one part of the boundary, the other part being for example inaccessible to measurements. Let us formulate the problem more precisely. Let $N, M \in \mathbb{N} \backslash\{0\}$ and $\Omega$ be a bounded open subset of $\mathbb{R}^{N}$ with boundary $\Gamma$ of class $C^{2}$. For a time $T>0$, we set $\mathcal{Q}=\Omega \times(0, T)$, $\Sigma=\Gamma \times(0, T)$, and $\Sigma_{0}=\Gamma_{0} \times(0, T)$, where $\Gamma_{0}$ is a non empty open subset of $\Gamma$. 
We consider the system modeling a problem of pollution [10]:

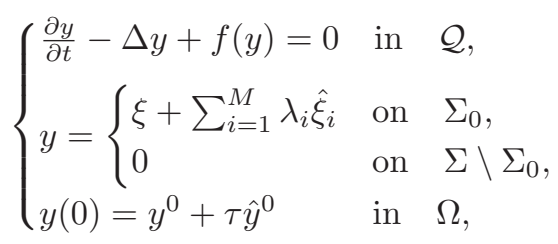

where:

- $y$ represents the concentration of the pollutant.

- $f$ is a given real function of class $\mathcal{C}^{1}$, globally Lipschitz.

- The boundary condition is unknown on a part $\Sigma_{0}$ of the boundary and represents here a pollution with a structure of the form $\xi+\sum_{i=1}^{M} \lambda_{i} \hat{\xi}_{i}$. In this structure, the functions $\xi$ and $\left\{\hat{\xi}_{i}\right\}_{i=1}^{M}$ are known whereas the reals $\left\{\lambda_{i}\right\}_{i=1}^{M}$ are unknown.

- The initial condition is unknown and its structure is of the form $y^{0}+\tau \hat{y}^{0}$ where the function $y^{0}$ is known and the term $\tau \hat{y}^{0}$ is unknown.

We set

$$
\begin{aligned}
H_{0}^{3 / 2}\left(\Gamma_{0}\right) & =\left\{\rho \in H^{3 / 2}\left(\Gamma_{0}\right) \text { such that } \rho=0 \text { on the boundary of } \Gamma_{0}\right\}, \\
\mathcal{H}\left(\Sigma_{0}\right) & =\left\{\rho \in H^{1}\left(0, T, H_{0}^{3 / 2}\left(\Gamma_{0}\right)\right) \text { such that } \rho(x, 0)=0 \text { on } \Gamma_{0}\right\}
\end{aligned}
$$

and we assume that:

- $y^{0}$ and $\hat{y}^{0}$ belong to $H_{0}^{1}(\Omega), \xi$ and $\hat{\xi}_{i}$ belong to $\mathcal{H}\left(\Sigma_{0}\right)$. Notice that if we write $\chi_{\Sigma_{0}}$ the characteristic function of the set $\Sigma_{0}$, this implies that $\xi \cdot \chi_{\Sigma_{0}}$ and $\hat{\xi}_{i} \cdot \chi_{\Sigma_{0}}$ are elements of $\mathcal{H}(\Sigma)$ where

$$
\mathcal{H}(\Sigma)=\left\{\rho \in H^{1}\left(0, T, H^{3 / 2}(\Gamma)\right) \text { such that } \rho(x, 0)=0 \text { on } \Gamma\right\} .
$$

- The functions

$$
\hat{\xi}_{i} \cdot \chi_{\Gamma_{0}}, \quad 1 \leq i \leq M, \quad \text { are linearly independent. }
$$

- The real $\tau$ is small enough and $\left\|\hat{y}^{0}\right\|_{H_{0}^{1}(\Omega)} \leq 1$.

- The function $f$ verifies (in order to simplify)

$$
f(0)=0 .
$$

For model (1), we are interested in identifying the parameters $\lambda_{i}$ without any attempt at computing $\tau \hat{y}^{0}$. To identify these parameters, we use the theory of sentinel in a general framework. First of all, let us recall that the usual theory of sentinels lies on three considerations: 
- A state equation represented here by (1) and where the solution $y=$ $y(x, t, \lambda, \tau)=y(\lambda, \tau)$ depends on two families of parameters $\lambda=\left\{\lambda_{1}, \cdots, \lambda_{M}\right\}$ and $\tau$.

- An observation $m_{0}$ which is a measure of the flux of the concentration of the pollutant taken on a non-empty open subset $O$ of $\Gamma \backslash \Gamma_{0}$ called observatory.

- A function $S$ called "sentinel," defined for $h_{0} \in L^{2}(O \times(0, T))$ by

$$
S(\lambda, \tau)=\int_{0}^{T} \int_{O}\left(h_{0}+w\right) \frac{\partial y}{\partial \nu} d x d t
$$

where $\nu$ is the unit exterior normal to $\Gamma, \frac{\partial y}{\partial \nu}$ is the derivative of $y$ with respect to the normal $\nu$ and $w$, the control function. Moreover, the control $w$ is to be found of minimal norm in $L^{2}(O \times(0, T))$ such that functions $S$ satisfy

- $S$ is stationary to the first order with respect to missing terms $\tau \hat{y}^{0}$ :

$$
\frac{\partial S}{\partial \tau}(0,0)=0 \quad \forall \hat{y}^{0},
$$

- $S$ is sensitive to the first order with respect to pollution terms $\lambda_{i} \xi_{i}$ :

$$
\frac{\partial S}{\partial \lambda_{i}}(0,0)=c_{i}, \quad 1 \leq i \leq M,
$$

where $c_{i}, 1 \leq i \leq M$, are given constants not all identically zero.

Using the adjoint problem, J.-L. Lions in [10] shows that the existence of these sentinels is reduced to solution of exact controllability problem with constraints on the state, but he only proved results of approximate controllability. These types of approximate controllability problems with constraints on the state were also the subject of many numerical resolutions. It is in this context, for instance that J. P. Kernevez et al. use these sentinels in $[1,7]$ to identify parameters of pollution in a river. O. Bodart apply them in [2] to identify an unknown boundary.

Remark. To estimate the parameter $\lambda_{i}$, one proceeds as follows:

Assume that the solution of (1) when $\lambda=0$ and $\tau=0$ is known. Then, one has the following information:

$$
S(\lambda, \tau)-S(0,0) \approx \sum_{i=1}^{M} \lambda_{i} \frac{\partial S}{\partial \lambda_{i}}(0,0) .
$$

Therefore, fixing $i \in\{1, \ldots, M\}$ and choosing

$$
\frac{\partial S}{\partial \lambda_{j}}(0,0)=0 \quad \text { for } \quad j \neq i \quad \text { and } \quad \frac{\partial S}{\partial \lambda_{i}}(0, \ldots, 0)=c_{i},
$$


one obtains the following estimate of the parameter $\lambda_{i}$ :

$$
\lambda_{i} \approx \frac{1}{c_{i}}(S(\lambda, \tau)-S(0,0)) .
$$

Remark. J. L. Lions refers to the function $S$ as a sentinel with given sensitivity $c_{i}$. In (7), the $c_{i}$ are chosen according to the importance which is conferred to the component $\xi_{i}$ of the pollution.

Remark. Notice that for the J. L. Lions's sentinels defined by (5)-(7), the observatory $O \subset\left(\Gamma \backslash \Gamma_{0}\right)$ is also the support of the control function $w$.

In this paper, we consider the general framework where the support of the control function $w$ may be different from the observatory $O$. More precisely, for any nonempty open subset $\gamma$ of $\Gamma \backslash \Gamma_{0}$ where $\gamma$ may be different from $O$, we look for a function $S=S(\lambda, \tau)$ solution of the following problem:

Given $h_{0} \in L^{2}(O \times(0, T))$, find $w \in L^{2}(\gamma \times(0, T))$ such that

(i) the function $S$ defined by

$$
S(\lambda, \tau)=\int_{0}^{T} \int_{O} h_{0} \frac{\partial y}{\partial \nu}(\lambda, \tau) d \Gamma d t+\int_{0}^{T} \int_{\gamma} w \frac{\partial y}{\partial \nu}(\lambda, \tau) d \Gamma d t
$$

satisfies the conditions (6) and (7);

(ii) The control $w$ is of minimal norm in $L^{2}(\gamma \times(0, T))$ among "the admissible controls," i.e.,

$$
|w|_{L^{2}(\gamma \times(0, T))}=\min _{\bar{w} \in E}|w|_{L^{2}(\gamma \times(0, T))}
$$

where

$$
E=\left\{\bar{w} \in L^{2}(\gamma \times(0, T)), \quad \text { such that }(\bar{w}, S(\bar{w})) \text { satisfies }(6)-(8)\right\} .
$$

Under the above hypotheses on $f$ and the data, one can prove as in [3] that there exists $\alpha>0$ such that when

$$
|\tau|+\sum_{i=1}^{M}\left|\lambda_{i}\right| \leq \alpha
$$

problem (1) admits a unique solution $y=y(\lambda, \tau)$ in

$$
H^{2,1}(\mathcal{Q})=L^{2}\left(0, T ; H^{2}(\Omega)\right) \cap H^{1}\left(0, T ; L^{2}(\Omega)\right) .
$$

Moreover, if we denote by $I \subset \mathbb{R}$ a neighborhood of 0 , the applications

$$
\tau \longmapsto y(\lambda, \tau) \quad \text { and } \quad \lambda_{i} \longmapsto y(\lambda, \tau) \quad(1 \leq i \leq M)
$$


are in $\mathcal{C}^{1}\left(I ; L^{2}\left(0, T ; H^{2}(\Omega)\right)\right)$. In fact, if we denote by $y_{0}=y(0,0) \in H^{2,1}(Q)$ the solution of (1) when $\lambda=0$ and $\tau=0$, and if we write the derivatives of $y$ at $(0,0)$ with respect to $\tau$ and $\lambda_{i}$ as

$$
y_{\tau}=\left.\frac{\partial}{\partial \tau} y(\lambda, \tau)\right|_{\tau=0, \lambda_{i}=0}
$$

and

$$
y_{\lambda_{i}}=\left.\frac{\partial}{\partial \lambda_{i}} y(\lambda, \tau)\right|_{\tau=0, \lambda_{i}=0}
$$

the functions $y_{\tau}$ and $y_{\lambda_{i}}$ are respectively solutions of

$$
\left\{\begin{aligned}
\frac{\partial y_{\tau}}{\partial t}-\Delta y_{\tau}+f^{\prime}\left(y_{0}\right) y_{\tau} & =0 & & \text { in } \mathcal{Q} \\
y_{\tau} & =0 & & \text { on } \Sigma \\
y_{\tau}(0) & =\hat{y}^{0} & & \text { in } \Omega
\end{aligned}\right.
$$

and

$$
\left\{\begin{aligned}
\frac{\partial y_{\lambda_{i}}}{\partial t}-\Delta y_{\lambda_{i}}+f^{\prime}\left(y_{0}\right) y_{\lambda_{i}} & =0 & & \text { in } \mathcal{Q}, \\
y_{\lambda_{i}} & =\hat{\xi}_{i} \chi_{\Sigma_{0}} & & \text { on } \Sigma \\
y_{\lambda_{i}}(0) & =0 & & \text { in } \Omega,
\end{aligned}\right.
$$

where $f^{\prime}\left(y_{0}\right)$ denotes the derivative of $f$ at point $y_{0}$. Since $f$ is a real $\mathcal{C}^{1}$ and globally Lipschitz function, we have

$$
a_{0}=f^{\prime}\left(y_{0}\right) \in L^{\infty}(\mathcal{Q})
$$

Consequently, $\hat{y}^{0}$ and $\hat{\xi}_{i}$ being respectively in $H_{0}^{1}(\Omega)$ and $\mathcal{H}\left(\Sigma_{0}\right)$, we deduce that problems (10) and (11) have respectively a unique solution $y_{\tau}$ and $y_{\lambda_{i}}$ in $H^{2,1}(\mathcal{Q})$.

Remark. Since $y \in L^{2}\left(0, T ; H^{2}(\Omega)\right)$, we have $\frac{\partial y}{\partial \nu} \in L^{2}\left(0, T ; H^{1 / 2}(\Gamma)\right) \subset L^{2}(\Sigma)$. Thus, $h_{0}$ and $w$ being respectively in $L^{2}(O \times(0, T))$ and in $L^{2}(\gamma \times(0, T))$, relation (8) is well defined. Moreover, $y_{\tau}$ and $y_{\lambda_{i}}$ belong to $L^{2}\left(0, T ; H^{2}(\Omega)\right)$. This means that $\frac{\partial y_{\tau}}{\partial \nu}$ and $\frac{\partial y_{\lambda_{i}}}{\partial \nu}$ belong to $L^{2}\left(0, T ; H^{1 / 2}(\Gamma)\right) \subset L^{2}(\Sigma)$. Thus, conditions (6) and (7) are well defined.

Let $\chi_{\gamma}$ be the characteristic function of the set $\gamma$. We set

$$
Y=\operatorname{Span}\left(\frac{\partial y_{\lambda_{1}}}{\partial \nu} \chi_{\gamma}, \ldots, \frac{\partial y_{\lambda_{M}}}{\partial \nu} \chi_{\gamma}\right)
$$


the vector subspace of $L^{2}(\gamma \times(0, T))$ generated by the $M$ functions $\frac{\partial y_{\lambda_{i}}}{\partial \nu} \chi_{\gamma}, 1 \leq i \leq M$, which will be proved to be independent (see Lemma 1.2 in subsection 1.1) and we denote by $Y^{\perp}$ the orthogonal of $Y$ in $L^{2}(\gamma \times(0, T))$. Let also

$$
Y_{\theta}=\frac{1}{\theta} Y
$$

be the vector subspace of $L^{2}(\gamma \times(0, T))$ generated by the $M$ functions $\frac{1}{\theta} \frac{\partial y_{\lambda_{i}}}{\partial \nu} \chi_{\gamma}$, $1 \leq i \leq M$, where $\theta$ is the positive function precisely defined later on by (26). Clearly, these functions will also be independent.

We consider the following controllability problem:

Given $h_{0} \in L^{2}(O \times(0, T)), w_{0} \in Y_{\theta}$, and $a_{0} \in L^{\infty}(\mathcal{Q})$, find $v \in L^{2}(\gamma \times(0, T))$ such that

$$
v \in Y^{\perp}
$$

and if $q=q(x, t, v) \in L^{2}(\mathcal{Q})$ is solution of

$$
\left\{\begin{aligned}
-\frac{\partial q}{\partial t}-\Delta q+a_{0} q & =0 & & \text { in } \mathcal{Q}, \\
q & =h_{0} \chi_{O}+\left(w_{0}-v\right) \chi_{\gamma} & & \text { on } \Sigma \\
q(T) & =0 & & \text { in } \Omega,
\end{aligned}\right.
$$

q satisfies

$$
q(x, 0, v)=0 \quad \text { in } \Omega .
$$

Remark. Let us notice that if $v$ exists, the set

$$
\mathcal{E}=\left\{\bar{v} \in Y^{\perp} \quad \text { such that } \quad(\bar{v}, \bar{q}=q(x, t, \bar{v})) \text { satisfies (15), (16) }\right\}
$$

is a non-empty, closed, and convex set in $L^{2}(\gamma \times(0, T))$. Therefore there exists $v \in \mathcal{E}$ of minimal norm.

The problem (14)-(16) is a null boundary controllability problem with constraint on the control. When $Y^{\perp}=L^{2}(\gamma \times(0, T))$, this problem becomes a null boundary controllability problem without constraint on the control. This problem has been studied by many authors.

In the linear case, D. Russell in [15] has proved that exact controllability for the wave equation implies exact controllability for the heat equation. Inspired by this work of D. Russell, G. Lebeau and L. Robbiano in [8] solved the problem of null boundary controllability in the case $a_{0}=0$ using observability inequalities deriving from Carleman inequalities. At the same time, O. Y. Imanuvilov and A. Fursikov in [5] obtained the same result for more general operators including variable coefficients and nonzero potentials using more directly global Carleman inequalities for the 
evolution operator. They could extend their method to the case of some nonlinear heat equations where they prove that the problem of null boundary controllability holds for sufficiently small initial data. We can also mention results in this direction by Y. J. Lin Guo and W. Littman in [9].

O. Nakoulima gives in [14] a result of null controllability for the heat equation with constraint on a distributed control. His results is based on an observability inequality adapted to the constraint.

In this paper we solve the null boundary controllability problem with constraint on the control (14)-(16). This allows us to prove the existence of the sentinel for given sensitivity (6)-(9). More precisely, we have the following results:

Theorem A. Let $\Omega$ be a bounded open subset of $\mathbb{R}^{N}$ with boundary $\Gamma$ of class $C^{2}$, $\Gamma_{0}$ a non-empty open subset of $\Gamma, O$ and $\gamma$ two non-empty open subsets of $\Gamma \backslash \Gamma_{0}$. Let also $f$ be real function of class $\mathcal{C}^{1}$, globally Lipschitz verifying (4). Then the existence of the sentinel (6)-(9) holds if and only if the null boundary controllability problem with constraint on the control (14)-(16) has a solution.

The proof of the boundary null-controllability problem with constraint on the control (14)-(16) lies on the existence of a function $\theta$ and a Carleman inequality adapted to the constraint (see subsection 2.1) for which we have the following result:

Theorem B. Assume that the hypotheses of Theorem A are satisfied. Then there exists a positive real weight function $\theta$ (a precise definition of $\theta$ will be given later on) such that, for any function $h_{0} \in L^{2}(O \times(0, T))$ with $\theta h_{0} \in L^{2}(O \times(0, T))$, there exists a unique control $\hat{v} \in L^{2}(\gamma \times(0, T))$ such that $(\hat{v}, \hat{q})$ with $\hat{q}=q(\hat{v})$ is solution of the null boundary controllability problem with constraint on the control (14)-(16) and provides a control $\hat{w}=w_{0} \chi_{\gamma}-\hat{v}$ of the sentinel problem satisfying (9).

Moreover, the control $\hat{w}$ is given by

$$
\hat{w}=P\left(w_{0}\right)+(I-P)\left(\frac{\partial \hat{\rho}}{\partial \nu} \chi_{\gamma}\right)
$$

where $P$ is the orthogonal projection operator from $L^{2}(\gamma \times(0, T))$ into $Y, w_{0} \in Y_{\theta}$ depends on $h_{0}$ and $c_{i}, i \in\{1, \ldots, M\}$, and will be precisely determined in (22), and $\hat{\rho}$ satisfies

$$
\left\{\begin{aligned}
\frac{\partial \hat{\rho}}{\partial t}-\Delta \hat{\rho}+a_{0} \hat{\rho} & =0 & & \text { in } \mathcal{Q} \\
\hat{\rho} & =0 & & \text { on } \Sigma .
\end{aligned}\right.
$$

The paper is organized as follows: Section 1 is devoted to the equivalence between the sentinel problem and the controllability problem with constraint on the control. In this section we give the proof of Theorem $\mathrm{A}$, the formulation of the sentinel and the estimate on the parameter $\lambda_{i}$. In section 2 , we study the null-controllability problem with constraint on the control (14)-(16) and prove Theorem B. 


\section{Equivalence between the sentinel problem and the control- lability problem with constraint on the control}

\subsection{Proof of Theorem A}

To obtain the boundary null-controllability problem (14)-(16), we interpret (6) and (7).

In view of (8), the stationary condition (6) and respectively the sensitivity conditions (7) hold if and only if

$$
\int_{0}^{T} \int_{O} h_{0} \frac{\partial y_{\tau}}{\partial \nu} d \Gamma d t+\int_{0}^{T} \int_{\gamma} w \frac{\partial y_{\tau}}{\partial \nu} d \Gamma d t=0 \quad \forall \hat{y}^{0} \in H_{0}^{1}(\Omega)
$$

and

$$
\int_{0}^{T} \int_{O} h_{0} \frac{\partial y_{\lambda_{i}}}{\partial \nu} d \Gamma d t+\int_{0}^{T} \int_{\gamma} w \frac{\partial y_{\lambda_{i}}}{\partial \nu} d \Gamma d t=c_{i}, \quad 1 \leq i \leq M
$$

Therefore, in order to transform equation (17), we introduce the classical adjoint state. More precisely, we consider the following linear problem

$$
\left\{\begin{aligned}
-\frac{\partial q}{\partial t}-\Delta q+a_{0} q & =0 & & \text { in } \mathcal{Q} \\
q & =h_{0} \chi_{O}+w \chi_{\gamma} & & \text { on } \Sigma \\
q(T) & =0 & & \text { in } \Omega .
\end{aligned}\right.
$$

Since $h_{0} \chi_{O}+w \chi_{\gamma} \in L^{2}(\Sigma)$, one can prove using transposition method that problem (19) admits a unique solution $q$ in $L^{2}(\mathcal{Q}) \cap \mathcal{C}\left([0, T], H^{-1}(\Omega)\right)$. Moreover we have $\frac{\partial q}{\partial \nu} \in H^{-1}\left(0, T ; H^{-3 / 2}(\Gamma)\right)$ (see $\left.[12]\right)$.

Remark 1.1. In fact, we have $\frac{\partial q}{\partial \nu} \in \mathcal{H}^{\prime}(\Sigma)$ where $\mathcal{H}(\Sigma)$ is defined in (2). In order to show this, we can take $T^{\prime}>T$, extend $h_{0}$ and $w$ by zero on $\left(T, T^{\prime}\right)$ and take $q\left(T^{\prime}\right)=0$ so that $q=0$ on $\left(T, T^{\prime}\right)$. Now if $\xi \in \mathcal{H}(\Sigma)$, we can extend it smoothly on $\left(T, T^{\prime}\right)$ so that $\xi\left(T^{\prime}\right)=0$. Therefore $\xi \in H_{0}^{1}\left(0, T^{\prime} ; H^{3 / 2}(\Gamma)\right)$ and the duality $\left\langle\frac{\partial q}{\partial \nu}, \xi\right\rangle_{H^{-1}\left(0, T^{\prime} ; H^{-3 / 2}(\Gamma)\right), H_{0}^{1}\left(0, T^{\prime} ; H^{3 / 2}(\Gamma)\right)}$ makes perfect sense with $\frac{\partial q}{\partial \nu}=0$ on $\left(T, T^{\prime}\right)$.

Now, multiplying both sides of the differential equation in (19) by $y_{\tau}$ solution of (10) and integrating by parts in $\mathcal{Q}$, we get for all $\hat{y}^{0} \in H_{0}^{1}(\Omega)$ that

$$
\int_{0}^{T} \int_{O} h_{0} \frac{\partial y_{\tau}}{\partial \nu} d \Gamma d t+\int_{0}^{T} \int_{\gamma} w \frac{\partial y_{\tau}}{\partial \nu} d \Gamma d t=-\left\langle q(0), \hat{y}^{0}\right\rangle_{H^{-1}(\Omega), H_{0}^{1}(\Omega)}
$$

where $\langle\cdot, \cdot\rangle_{X, X^{\prime}}$ represents now and in the sequel, the duality bracket between the space $X$ and $X^{\prime}$.

Thus, condition (6) (or (17)) holds if and only if

$$
q(0)=0 \quad \text { in } \Omega
$$


Then, multiplying both sides of the differential equation in (19) by $y_{\lambda_{i}}$ solution of (11) and integrating by parts in $\mathcal{Q}$, we have

$$
\left\langle\frac{\partial q}{\partial \nu}, \hat{\xi}_{i} \chi_{\Gamma_{0}}\right\rangle_{\mathcal{H}^{\prime}(\Sigma), \mathcal{H}(\Sigma)}=\int_{0}^{T} \int_{O} h_{0} \frac{\partial y_{\lambda_{i}}}{\partial \nu} d \Gamma d t+\int_{0}^{T} \int_{\gamma} w \frac{\partial y_{\lambda_{i}}}{\partial \nu} d \Gamma d t, \quad 1 \leq i \leq M .
$$

Thus, condition (7) (or (18)) is equivalent to

$$
\left\langle\frac{\partial q}{\partial \nu}, \hat{\xi}_{i} \chi_{\Gamma_{0}}\right\rangle_{\mathcal{H}^{\prime}(\Sigma), \mathcal{H}(\Sigma)}=c_{i}, \quad 1 \leq i \leq M
$$

The above considerations show that the existence of the sentinel of given sensitivity (6)-(9) is reduced to the solution of the following null controllability problem:

Given $h_{0} \in L^{2}(O \times(0, T))$ and $a_{0} \in L^{\infty}(\mathcal{Q})$, find the control $w$ of minimal norm in $L^{2}(\gamma \times(0, T))$ such that the pair $(w, q)$ is solution of $(19)-(21)$.

This new problem is a null-controllability problem with constraints (21) on the state $q$. Actually, let us show that the insensitivity conditions (7) (or the contraints (21)) on the state $q$ are equivalent to constraint on the control.

Lemma 1.2. Assume that (3) holds. Then the functions $\frac{\partial y_{\lambda_{i}}}{\partial \nu} \chi_{\gamma}, 1 \leq i \leq M$, are linearly independent. Moreover the functions $\frac{1}{\theta} \frac{\partial y_{\lambda_{i}}}{\partial \nu} \chi_{\gamma}, 1 \leq i \leq M$, are also linearly independent.

Proof. Let $\alpha_{i} \in \mathbb{R}, 1 \leq i \leq M$ be such that $\sum_{1=1}^{M} \alpha_{i} \frac{\partial y_{\lambda_{i}}}{\partial \nu} \chi_{\gamma}=0$. Then, in view of (11), $k=\sum_{1=1}^{M} \alpha_{i} y_{\lambda_{i}}$ is such that

$$
\left\{\begin{aligned}
\frac{\partial k}{\partial t}-\Delta k+f^{\prime}\left(y_{0}\right) k & =0 & & \text { in } \mathcal{Q}, \\
k & =\sum_{1=1}^{M} \alpha_{i} \hat{\xi}_{i} \chi_{\Sigma_{0}} & & \text { on } \Sigma, \\
\frac{\partial k}{\partial \nu} & =0 & & \text { on } \gamma \times(0, T), \\
k(0) & =0 & & \text { in } \Omega .
\end{aligned}\right.
$$

As $\gamma \subset \Gamma \backslash \Gamma_{0}$, we have $k=0$ and $\frac{\partial k}{\partial \nu}=0$ on $\gamma \times(0, T)$. Thanks to the unique continuation property $([13,16])$, this implies $k=0$ in $\mathcal{Q}$. Therefore, we deduce that $\sum_{1=1}^{M} \alpha_{i} \hat{\xi}_{i}=0$ on $\Gamma_{0} \times(0, T)$. Hence, assumption (3) allows to conclude that $\alpha_{i}=0$ for $1 \leq i \leq M$.

The second assertion of the lemma follows immediately.

Now the matrix

$$
\left(\int_{0}^{T} \int_{\gamma} \frac{1}{\theta} \frac{\partial y_{\lambda_{j}}}{\partial \nu} \frac{\partial y_{\lambda_{i}}}{\partial \nu} d \Gamma d t\right)_{i, j}
$$


is symmetric positive definite and, therefore, there exists a unique $w_{0} \in Y_{\theta}$ such that

$$
c_{i}-\int_{0}^{T} \int_{O} h_{0} \frac{\partial y_{\lambda_{i}}}{\partial \nu} d \Gamma d t=\int_{0}^{T} \int_{\gamma} w_{0} \frac{\partial y_{\lambda_{i}}}{\partial \nu} d \Gamma d t, \quad 1 \leq i \leq M .
$$

Consequently, combining (18) with (22), we observe that condition (7) (or the contraints (21)) holds if and only if

$$
w-w_{0}=-v \in Y^{\perp},
$$

where $Y$ is given by (13). Replacing $w$ by $-v+w_{0}$ in the second expression of (19), we obtain the second one in (15). Therefore, the sentinel problem (6)-(9) holds if and only if null boundary controllability problem with constraint on the control (14)-(16) has a solution.

Remark 1.3. The function $w_{0}$ is such that $\theta w_{0} \in L^{2}(\gamma \times(0, T))$. The choice of $w_{0}$ in $Y_{\theta}$ will be necessary for the construction of the optimal control for the null boundary controllability problem (14)-(16) in section 2 .

Remark 1.4. If $\mathcal{E}$ is the set of admissible controls $v \in L^{2}(\gamma \times(0, T))$ such that (14)-(16) is satisfied, then $\mathcal{E}$ is a closed convex subset of $L^{2}(\gamma \times(0, T))$. Since $w_{0}-\mathcal{E}$ is also a closed convex subset of $L^{2}(\gamma \times(0, T))$, we can obtain $w$ to be of minimal norm in $L^{2}(\gamma \times(0, T))$ by minimizing the norm of $w_{0}-v$ when $v \in \mathcal{E}$. Then the pair $(v, q(v))$ satisfying (14)-(16) necessarily provides a control $w$ satisfying (9).

\subsection{Formulation of the sentinel with given sensitivity and identification of parameter $\lambda_{i}$}

Assume that Theorem B holds. Then if we replace in (8) w with

$$
\hat{w}=P\left(w_{0}\right)+(I-P) \frac{\partial \hat{\rho}}{\partial \nu} \chi_{\gamma},
$$

the function $S$ defined by

$$
\begin{aligned}
S(\lambda, \tau)=\int_{0}^{T} \int_{O} h_{0} \frac{\partial y}{\partial \nu}(\lambda, \tau) d \Gamma & d t \\
& +\int_{0}^{T} \int_{\gamma}\left(P\left(w_{0}\right)+(I-P) \frac{\partial \hat{\rho}_{\theta}}{\partial \nu} \chi_{\gamma}\right) \frac{\partial y}{\partial \nu}(\lambda, \tau) d \Gamma d t
\end{aligned}
$$

is such that $(\hat{w}, S)$ verifies $(6)-(9)$. Therefore proceeding as in the remark in page 167 , we deduce that

$$
\begin{aligned}
\lambda_{i} \approx \frac{1}{c_{i}}\left\{\int_{0}^{T} \int_{O} h_{0}\left(m_{0}-\frac{\partial y_{0}}{\partial \nu}\right) d \Gamma d t\right\} \\
+\frac{1}{c_{i}}\left\{\int_{0}^{T} \int_{\gamma}\left(P\left(w_{0}\right)+(I-P) \frac{\partial \hat{\rho}_{\theta}}{\partial \nu} \chi_{\gamma}\right)\left(m_{0}-\frac{\partial y_{0}}{\partial \nu}\right) d \Gamma d t\right\},
\end{aligned}
$$


where $m_{0}$ is a measure of the flux of the concentration of the pollutant taken on the observatory $O \cup \gamma$ and $y_{0}$ is solution of (1) when $\lambda=0$ and $\tau=0$.

\section{Study of the boundary null-controllability problem with con- straint on the control}

In this section, we prove existence of the solution of the boundary null controllability problem (14)-(16) and of course uniqueness if we want the control to be of minimal norm among admissible controls. The main tool we use is an observability inequality adapted to the constraint (14) which itself will be a consequence of an adapted Carleman inequality.

\subsection{An adapted Carleman inequality}

Let us consider an auxiliary function $\psi \in C^{2}(\bar{\Omega})$ which satisfies the following conditions:

$$
\begin{aligned}
\Psi(x)>0 & \forall x \in \Omega, \\
|\nabla \Psi|>\alpha>0 & \forall x \in \bar{\Omega}, \\
\Psi(x)=0 & \text { on } \Gamma \backslash \gamma, \\
\frac{\partial \Psi}{\partial \nu}(x)<0 & \forall x \in \Gamma \backslash \gamma .
\end{aligned}
$$

Such a function $\psi$ exists according to A. Fursikov and O. Yu. Imanuvilov [5].

For $(x, t) \in \mathcal{Q}$, we define for any positive parameter value $\lambda$ the following weight functions:

$$
\begin{aligned}
\varphi(x, t) & =\frac{\mathrm{e}^{\lambda\left(m|\Psi|_{\infty}+\Psi(x)\right)}}{t(T-t)}, \\
\eta(x, t) & =\frac{\mathrm{e}^{2 \lambda m|\Psi|_{\infty}}-\mathrm{e}^{\lambda\left(m|\Psi|_{\infty}+\Psi(x)\right)}}{t(T-t)}
\end{aligned}
$$

with $m>1$. Weight functions of this kind were first introduced by O. Yu. Imanuvilov. Since $\varphi$ does not vanish in $\mathcal{Q}$, for all $s>0$ and $\lambda>0$, we set

$$
\frac{1}{\theta^{2}}=\min \left[e^{-2 s \eta}\left(\varphi^{-1}, \varphi, \varphi^{3}, \varphi\left|\frac{\partial \Psi}{\partial \nu}\right|\right)\right]
$$

and we adopt the following notations:

$$
\begin{aligned}
L & =\frac{\partial}{\partial t}-\Delta+a_{0} I, \\
L^{*} & =-\frac{\partial}{\partial t}-\Delta+a_{0} I, \\
\mathcal{V} & =\left\{\rho \in C^{\infty}(\bar{Q}), \quad \rho=0 \text { on } \Sigma\right\},
\end{aligned}
$$


where $a_{0} \in L^{\infty}(\mathcal{Q})$ is defined by (12). Then, using the notations given by (27), we have the following inequality:

Proposition 2.1 (Adapted Carleman inequality). Assume that (3) holds. Let $Y$ be the real vector subspace of $L^{2}(\gamma \times(0, T))$ of finite dimension defined in (13) and $P$ be the orthogonal projection operator from $L^{2}(\gamma \times(0, T))$ into $Y$. Let also $\theta$ be the function defined by (26). Then, there exist numbers $\lambda_{0}=\lambda_{0}\left(\Omega, \gamma, a_{0}\right)>1, s_{0}=$ $s_{0}\left(\Omega, \gamma, a_{0}, T\right)>1, C=C\left(\Omega, \gamma, a_{0}\right)>0$ such that, for fixed $\lambda \geq \lambda_{0}$ and $s \geq s_{0}$ and for any $\rho \in \mathcal{V}$,

$$
\int_{0}^{T} \int_{\Gamma} \frac{1}{\theta^{2}}\left|\frac{\partial \rho}{\partial \nu}\right|^{2} d \Gamma d t \leq C\left[\int_{0}^{T} \int_{\Omega}|L \rho|^{2} d x d t+\int_{0}^{T} \int_{\gamma}\left|P \frac{\partial \rho}{\partial \nu}-\frac{\partial \rho}{\partial \nu} \chi_{\gamma}\right|^{2} d \Gamma d t\right] .
$$

To prove Proposition 2.1, we need some preliminary results.

Proposition 2.2 (Boundary Carleman inequality). Let $\Psi, \varphi$ and $\eta$ be the functions defined respectively by (23)-(25). Then, there exist numbers $\lambda_{0}=\lambda_{0}\left(\Omega, \gamma, a_{0}\right)>1$, $s_{0}=s_{0}\left(\Omega, \gamma, a_{0}, T\right)>1, C_{0}=C_{0}\left(\Omega, \gamma, a_{0}\right)>0$, and $C_{1}=C_{1}\left(\Omega, \gamma, a_{0}\right)>0$ such that, for any $\lambda \geq \lambda_{0}$, for any $s \geq s_{0}$, and for any $\rho \in \mathcal{V}$, the following estimate holds:

$$
\begin{gathered}
\int_{0}^{T} \int_{\Omega} e^{-2 s \eta}\left((s \varphi)^{-1}\left(\left|\frac{\partial \rho}{\partial t}\right|^{2}+|\Delta \rho|^{2}\right)+s \lambda^{2} \varphi|\nabla \rho|^{2}+s^{3} \lambda^{4} \varphi^{3}|\rho|^{2}\right) d x d t \\
+C_{0} \int_{0}^{T} \int_{\Gamma \backslash \gamma} s e^{-2 s \eta} \varphi\left(-\frac{\partial \Psi}{\partial \nu}\right)\left|\frac{\partial \rho}{\partial \nu}\right|^{2} d \Gamma d t \\
\leq C_{1}\left[\int_{0}^{T} \int_{\Omega} e^{-2 s \eta}|L \rho|^{2} d x d t+\int_{0}^{T} \int_{\gamma} s e^{-2 s \eta} \varphi\left|\frac{\partial \rho}{\partial \nu}\right|^{2} d \Gamma d t\right] .
\end{gathered}
$$

Proof. See $[4,6]$.

As a consequence of Proposition 2.2, we have this other inequality:

Proposition 2.3. Let $\theta$ be defined as in (26). Then, there exist numbers $\lambda_{0}=\lambda_{0}\left(\Omega, \gamma, a_{0}\right)>1, s_{0}=s_{0}\left(\Omega, \gamma, a_{0}, T\right)>1, C_{0}=C_{0}\left(\Omega, \gamma, a_{0}\right)>0$, and $C_{1}=C_{1}\left(\Omega, \gamma, a_{0}\right)>0$ such that, for fixed $\lambda \geq \lambda_{0}$ and $s \geq s_{0}$ and for any $\rho \in \mathcal{V}$,

$$
\begin{array}{r}
\int_{0}^{T} \int_{\Omega} \frac{1}{\theta^{2}}\left(\left|\frac{\partial \rho}{\partial t}\right|^{2}+|\Delta \rho|^{2}+|\nabla \rho|^{2}+|\rho|^{2}\right) d x d t+C_{0} \int_{0}^{T} \int_{\Gamma} \frac{1}{\theta^{2}}\left|\frac{\partial \rho}{\partial \nu}\right|^{2} d \Gamma d t \\
\leq C_{1}\left[\int_{0}^{T} \int_{\Omega}|L \rho|^{2} d x d t+\int_{0}^{T} \int_{\gamma}\left|\frac{\partial \rho}{\partial \nu}\right|^{2} d \Gamma d t\right] .
\end{array}
$$

Proof. As $\Psi$ belongs to $\mathcal{C}^{2}(\bar{\Omega})$ and $\varphi e^{-2 s \eta}$ is bounded, it is immediate that $\frac{1}{\theta}$ is also bounded in $\mathcal{Q}$. Consequently, adding the term $\int_{0}^{T} \int_{\gamma} s \varphi e^{-2 s \eta}\left|\frac{\partial \Psi}{\partial \nu}\right|\left|\frac{\partial \rho}{\partial \nu}\right|^{2} d \Gamma d t$ to each side of (29), then using the property of $\Psi$ given by the fourth condition in (23) and the fact that $s>1$ and $\lambda>1$, we deduce (30). 
Lemma 2.4. Assume that (3) holds. Let $Y$ be defined by (13). Then any function $\rho$ such that

$$
\left\{\begin{array}{rlrl}
\frac{\partial \rho}{\partial t}-\Delta \rho+a_{0} \rho & =0 & & \text { in } \mathcal{Q}, \\
\rho & =0 & & \text { on } \Sigma, \\
\left.\frac{\partial \rho}{\partial \nu}\right|_{\gamma} & \in Y &
\end{array}\right.
$$

is identically zero.

Proof. For any $\rho$ verifying (31), there exists $\alpha_{i} \in \mathbb{R}, 1 \leq i \leq M$, such that $\frac{\partial \rho}{\partial \nu}=\sum_{i=1}^{M} \alpha_{i} \frac{\partial y_{\lambda_{i}}}{\partial \nu}$ on $\gamma$. We set $z=\rho-\sum_{i=1}^{M} \alpha_{i} y_{\lambda_{i}}$. Then in view of (11), we have

$$
\left\{\begin{aligned}
\frac{\partial z}{\partial t}-\Delta z+a_{0} z & =0 & & \text { in } \mathcal{Q} \\
z & =\sum_{i=1}^{M} \alpha_{i} \hat{\xi}_{i} \chi_{\Sigma_{0}} & & \text { on } \Sigma \\
\frac{\partial z}{\partial \nu} & =0 & & \text { on } \gamma \times(0, T) .
\end{aligned}\right.
$$

As $\gamma \subset \Gamma \backslash \Gamma_{0}$, we have

$$
z=0 \quad \text { and } \quad \frac{\partial z}{\partial \nu}=0 \quad \text { on } \quad \gamma \times(0, T) .
$$

Therefore, thanks to the unique continuation property (see $[13,16]$ ), we have $z=0$ in $\mathcal{Q}$. Consequently, we deduce on the one hand that $\rho=\sum_{i=1}^{M} \alpha_{i} y_{\lambda_{i}}$ and on the other hand that $\sum_{i=1}^{M} \alpha_{i} \hat{\xi}_{i}=0$ on $\Sigma_{0}$. Hence, it follows from assumption (3) that $\alpha_{i}=0$ for $1 \leq i \leq M$. Thus, $\rho=0$ in $\mathcal{Q}$.

Proof of Proposition 2.1. The proof uses a well known compactness-uniqueness argument and inequality (30). We argue by contradiction. We suppose that for any $n \in \mathbb{N}$ there exists $\rho_{n} \in \mathcal{V}$ such that

$$
\begin{array}{r}
\int_{0}^{T} \int_{\Omega}\left|L \rho_{n}\right|^{2} d x d t \leq \frac{1}{n} \\
\int_{0}^{T} \int_{\gamma}\left|P \frac{\partial \rho_{n}}{\partial \nu}-\frac{\partial \rho_{n}}{\partial \nu} \chi_{\gamma}\right|^{2} d \Gamma d t \leq \frac{1}{n}
\end{array}
$$

and

$$
\int_{0}^{T} \int_{\Gamma} \frac{1}{\theta^{2}}\left|\frac{\partial \rho_{n}}{\partial \nu}\right|^{2} d \Gamma d t=1
$$

Now let us prove that (32)-(34) yield a contradiction. We do it in three steps. 
- Step 1. We have

$$
\begin{array}{rl}
\int_{0}^{T} \int_{\gamma} \frac{1}{\theta^{2}}\left|P \frac{\partial \rho_{n}}{\partial \nu}\right|^{2} & d \Gamma d t \\
& \leq 2 \int_{0}^{T} \int_{\gamma} \frac{1}{\theta^{2}}\left|P \frac{\partial \rho_{n}}{\partial \nu}-\frac{\partial \rho_{n}}{\partial \nu}\right|^{2} d \Gamma d t+2 \int_{0}^{T} \int_{\gamma} \frac{1}{\theta^{2}}\left|\frac{\partial \rho_{n}}{\partial \nu}\right|^{2} d \Gamma d t
\end{array}
$$

Since the function $\frac{1}{\theta^{2}}$ is bounded in $\mathcal{Q}$, using (33) and (34), we conclude that there exists a constant $C$ independent of $n$ such that

$$
\int_{0}^{T} \int_{\gamma} \frac{1}{\theta^{2}}\left|P \frac{\partial \rho_{n}}{\partial \nu}\right|^{2} d \Gamma d t \leq C
$$

Therefore, $Y$ being a finite dimensional vector subspace of $L^{2}(\gamma \times(0, T))$, we deduce that

$$
\left|P \frac{\partial \rho_{n}}{\partial \nu}\right|_{L^{2}(\gamma \times(0, T))} \leq C .
$$

Consequently, using (33) and (35), we get

$$
\left|\frac{\partial \rho_{n}}{\partial \nu}\right|_{L^{2}(\gamma \times(0, T))} \leq C .
$$

- Step 2. Let us define $L^{2}\left(\frac{1}{\theta}, X\right)=\left\{\varphi \in L^{2}(X), \int_{X} \frac{1}{\theta^{2}}|\varphi|^{2} d X<\infty\right\}$. Then $L^{2}(X) \subset L^{2}\left(\frac{1}{\theta}, X\right)$ and the canonical injection is continuous. Thus, according to (32) and (36), we deduce from (30) that $\rho_{n}, \nabla \rho_{n}, \Delta \rho_{n}$ are bounded in $L^{2}\left(\frac{1}{\theta}, \mathcal{Q}\right)$ and $\frac{\partial \rho_{n}}{\partial \nu}$ is bounded in $L^{2}\left(\frac{1}{\theta}, \Sigma\right)$. Let us then take a subsequence still denoted by $\left(\rho_{n}\right)$ such that

$$
\left(\rho_{n}\right) \longrightarrow \rho \text { weakly in } L^{2}\left(\frac{1}{\theta}, \mathcal{Q}\right) \text {. }
$$

If we refer to (24) and (25) and the definition of $\frac{1}{\theta}$ given by (26), we can see that $\left(\rho_{n}\right)$ is bounded in $L^{2}(] \beta, T-\beta\left[; H^{2}(\Omega)\right), \forall \beta>0$. Then, we have in particular, for every $\beta>0$,

$$
\begin{array}{cc}
\rho_{n} \longrightarrow \rho & \text { weakly in } L^{2}(] \beta, T-\beta[\times \Omega), \\
\frac{\partial \rho_{n}}{\partial \nu} \longrightarrow \frac{\partial \rho}{\partial \nu} & \text { weakly in } L^{2}(] \beta, T-\beta[\times \Gamma) .
\end{array}
$$

This implies that

$$
\begin{array}{cc}
\rho_{n} \longrightarrow \rho & \text { weakly in } D^{\prime}(\mathcal{Q}), \\
\frac{\partial \rho_{n}}{\partial \nu} \longrightarrow \frac{\partial \rho}{\partial \nu} & \text { weakly in } D^{\prime}(\Sigma) .
\end{array}
$$


Therefore, we get from (32) and (36) that

$$
\begin{array}{ll}
L \rho_{n} \longrightarrow L \rho=0 & \text { strongly in } L^{2}(\mathcal{Q}), \\
\frac{\partial \rho_{n}}{\partial \nu} \longrightarrow \frac{\partial \rho}{\partial \nu} & \text { weakly in } L^{2}(\gamma \times(0, T)) .
\end{array}
$$

And, since $P$ is a compact operator, we deduce from (38) that

$$
P \frac{\partial \rho_{n}}{\partial \nu} \longrightarrow P \frac{\partial \rho}{\partial \nu} \quad \text { strongly in } L^{2}(\gamma \times(0, T)) .
$$

From (33), we also have

$$
P \frac{\partial \rho_{n}}{\partial \nu}-\frac{\partial \rho_{n}}{\partial \nu} \chi_{\gamma} \longrightarrow 0 \quad \text { strongly in } L^{2}(\gamma \times(0, T))
$$

Thus, combining (39) with (40), we obtain

$$
\frac{\partial \rho_{n}}{\partial \nu} \chi_{\gamma} \longrightarrow P \frac{\partial \rho}{\partial \nu} \quad \text { strongly in } L^{2}(\gamma \times(0, T))
$$

Thanks to the uniqueness of the limit in $L^{2}(\gamma \times(0, T))$, the convergence relations (38) and (41) imply $P \frac{\partial \rho}{\partial \nu}=\frac{\partial \rho}{\partial \nu} \chi_{\gamma}$. This means that $\frac{\partial \rho}{\partial \nu} \chi_{\gamma} \in Y$. In short, we have proved that $\rho$ verifies

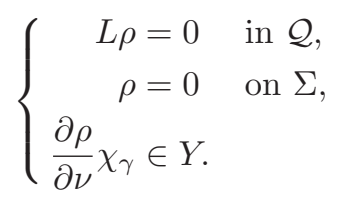

Thanks to Lemma $2.4, \rho$ is identically zero. Thus, $\frac{\partial \rho}{\partial \nu}=0$ on $\gamma \times(0, T)$ and (41) becomes

$$
\frac{\partial \rho_{n}}{\partial \nu} \longrightarrow 0 \quad \text { strongly in } L^{2}(\gamma \times(0, T)) .
$$

- Step 3. Since $\left(\rho_{n}\right) \in \mathcal{V}$, applying Carleman inequality (30) to $\rho_{n}$, then, passing to the limit on $n$, it results from (37) and (42) that $\frac{1}{\theta} \frac{\partial \rho_{n}}{\partial \nu} \rightarrow 0$ strongly in $L^{2}(\Sigma)$. Therefore, the contradiction occurs with (34).

\subsection{Solution of the boundary null-controllability problem with constraints on the control}

The proof of Theorem B can be obtained in different ways, all of them using the adapted Carleman estimate which has been proved in the previous section. Here we choose to present a proof which uses a penalization argument and which will be divided in three steps. 
- Step 1. For every $\varepsilon>0$ let us consider the following optimal control problem. Let $w_{0}$ be defined by (22). If $v \in Y^{\perp}$ let $q$ be solution of (15). We know that $q \in C\left([0, T] ; H^{-1}(\Omega)\right)$ and we can define the functional

$$
J_{\varepsilon}(v)=\frac{1}{2}\left|w_{0}-v\right|_{L^{2}(\gamma \times(0, T))}^{2}+\frac{1}{2 \varepsilon}|q(0)|_{H^{-1}(\Omega)}^{2}
$$

with $\left.|\varphi|_{H^{-1}(\Omega)}^{2}=\langle-\Delta)^{-1}, \varphi\right\rangle_{H_{0}^{1}(\Omega), H^{-1}(\Omega)}$ where $(-\Delta)^{-1} \varphi=\psi$ satisfies $-\Delta \psi=\varphi$ in $\Omega, \psi=0$ on $\Gamma$.

The optimal control problem is then to find $v_{\varepsilon} \in Y^{\perp}$ such that

$$
J_{\varepsilon}\left(v_{\varepsilon}\right)=\min _{v \in Y^{\perp}} J_{\varepsilon}(v) .
$$

As $Y^{\perp}$ is closed and convex (it is a linear subspace), it is classical to show that there exists a unique solution $v_{\varepsilon}$ to (43) (see for example [11]). If we write $q_{\varepsilon}$ the solution to (15) corresponding to $v_{\varepsilon}$, using an adjoint state $\rho_{\varepsilon}$, the triple $\left(q_{\varepsilon}, \rho_{\varepsilon}, v_{\varepsilon}\right)$ is solution of the following first order optimality system

$$
\begin{aligned}
& \left\{\begin{aligned}
L^{*} q_{\varepsilon} & =0 & & \text { in } \quad \mathcal{Q}, \\
q_{\varepsilon} & =h_{0} \chi_{O}+w_{0} \chi_{\gamma}-v_{\varepsilon} \chi_{\gamma} & & \text { on } \Sigma, \\
q_{\varepsilon}(T) & =0 & & \text { in } \Omega,
\end{aligned}\right. \\
& \left\{\begin{aligned}
L \rho_{\varepsilon} & =0 & & \text { in } \quad \mathcal{Q}, \\
\rho_{\varepsilon} & =0 & & \text { on } \Sigma, \\
\rho_{\varepsilon}(0) & =(-\Delta)^{-1}\left(\frac{1}{\varepsilon} q_{\varepsilon}(0)\right) & & \text { in } \quad \Omega,
\end{aligned}\right. \\
& v_{\varepsilon}=\left(w_{0} \chi_{\gamma}-\frac{\partial \rho_{\varepsilon}}{\partial \nu} \chi_{\gamma}\right)-P\left(w_{0} \chi_{\gamma}-\frac{\partial \rho_{\varepsilon}}{\partial \nu} \chi_{\gamma}\right) \in Y^{\perp} .
\end{aligned}
$$

- Step 2. We will here obtain estimates independent of $\varepsilon$ on the control $v_{\varepsilon}$ and on the state and adjoint state $q_{\varepsilon}$ and $\rho_{\varepsilon}$.

In view of the value of $v_{\varepsilon}$ given in (46), in order to obtain bounds for $v_{\varepsilon}$ it is enough to obtain bounds for $\left(\frac{\partial \rho_{\varepsilon}}{\partial \nu} \chi_{\gamma}-P \frac{\partial \rho_{\varepsilon}}{\partial \nu} \chi_{\gamma}\right)$.

Let us multiply the state equation (44) by $\rho_{\varepsilon}$. We obtain

$$
\left\langle\rho_{\varepsilon}(0), q_{\varepsilon}(0)\right\rangle_{H_{0}^{1}(\Omega), H^{-1}(\Omega)}+\int_{0}^{T} \int_{\Gamma} q_{\varepsilon} \frac{\partial \rho_{\varepsilon}}{\partial \nu} d \Gamma d t=0
$$

and therefore

$$
\frac{1}{\varepsilon}\left|q_{\varepsilon}(0)\right|_{H^{-1}(\Omega)}^{2}+\int_{0}^{T} \int_{\gamma}\left(w_{0}-v_{\varepsilon}\right) \frac{\partial \rho_{\varepsilon}}{\partial \nu} d \Gamma d t+\int_{0}^{T} \int_{O} h_{0} \frac{\partial \rho_{\varepsilon}}{\partial \nu} d \Gamma d t=0,
$$


so that

$$
\begin{array}{rl}
\frac{1}{\varepsilon}\left|q_{\varepsilon}(0)\right|_{H^{-1}(\Omega)}^{2}+\int_{0}^{T} \int_{\gamma}\left|w_{0}-v_{\varepsilon}\right|^{2} & d \Gamma d t+\int_{0}^{T} \int_{O} h_{0} \frac{\partial \rho_{\varepsilon}}{\partial \nu} d \Gamma d t \\
& -\int_{0}^{T} \int_{\gamma}\left(w_{0}-v_{\varepsilon}\right) P\left(w_{0} \chi_{\gamma}-\frac{\partial \rho_{\varepsilon}}{\partial \nu} \chi_{\gamma}\right) d \Gamma d t=0 .
\end{array}
$$

As $v_{\varepsilon} \in Y^{\perp}$, this gives

$$
\begin{aligned}
2 J_{\varepsilon}\left(v_{\varepsilon}\right)=\int_{0}^{T} \int_{\gamma}\left|P w_{0} \chi_{\gamma}\right|^{2} d \Gamma d t & +\int_{0}^{T} \int_{\gamma} w_{0}(I-P)\left(\frac{\partial \rho_{\varepsilon}}{\partial \nu} \chi_{\gamma}\right) d \Gamma d t \\
& -\int_{0}^{T} \int_{\gamma} w_{0} \frac{\partial \rho_{\varepsilon}}{\partial \nu} \chi_{\gamma} d \Gamma d t-\int_{0}^{T} \int_{O} h_{0} \frac{\partial \rho_{\varepsilon}}{\partial \nu} \chi_{\gamma} d \Gamma d t
\end{aligned}
$$

As we have on $\gamma \times(0, T)$

$$
w_{0}-v_{\varepsilon}=P w_{0} \chi_{\gamma}+(I-P)\left(\frac{\partial \rho_{\varepsilon}}{\partial \nu} \chi_{\gamma}\right)
$$

we have

$$
\int_{0}^{T} \int_{\gamma}\left|w_{0}-v_{\varepsilon}\right|^{2} d \Gamma d t=\int_{0}^{T} \int_{\gamma}\left|P w_{0} \chi_{\gamma}\right|^{2} d \Gamma d t+\int_{0}^{T} \int_{\gamma}\left|(I-P)\left(\frac{\partial \rho_{\varepsilon}}{\partial \nu} \chi_{\gamma}\right)\right|^{2} d \Gamma d t
$$

and we obtain

$$
\begin{aligned}
\frac{1}{\varepsilon}\left|q_{\varepsilon}(0)\right|_{H^{-1}(\Omega)}^{2}+\int_{0}^{T} \int_{\gamma} \mid & \left.(I-P)\left(\frac{\partial \rho_{\varepsilon}}{\partial \nu} \chi_{\gamma}\right)\right|^{2} d \Gamma d t \\
= & \int_{0}^{T} \int_{\gamma} w_{0}(I-P)\left(\frac{\partial \rho_{\varepsilon}}{\partial \nu} \chi_{\gamma}\right) d \Gamma d t \\
& \quad-\int_{0}^{T} \int_{\gamma} w_{0} \frac{\partial \rho_{\varepsilon}}{\partial \nu} \chi_{\gamma} d \Gamma d t-\int_{0}^{T} \int_{O} h_{0} \frac{\partial \rho_{\varepsilon}}{\partial \nu} \chi_{\gamma} d \Gamma d t
\end{aligned}
$$

This implies

$$
\begin{aligned}
& \frac{1}{\varepsilon}\left|q_{\varepsilon}(0)\right|_{H^{-1}(\Omega)}^{2}+\frac{1}{2} \int_{0}^{T} \int_{\gamma}\left|(I-P)\left(\frac{\partial \rho_{\varepsilon}}{\partial \nu} \chi_{\gamma}\right)\right|^{2} d \Gamma d t \\
& \leq \frac{1}{2} \int_{0}^{T} \int_{\gamma}\left|w_{0}\right|^{2} d \Gamma d t+\left(\int_{0}^{T} \int_{\gamma} \theta^{2}\left|w_{0}\right|^{2} d \Gamma d t+\int_{0}^{T} \int_{O} \theta^{2}\left|h_{0}\right|^{2} d \Gamma d t\right)^{1 / 2} \\
& \times\left(\int_{0}^{T} \int_{\Gamma} \frac{1}{\theta^{2}}\left|\frac{\partial \rho_{\varepsilon}}{\partial \nu}\right|^{2} d \Gamma d t\right)^{1 / 2}
\end{aligned}
$$


Now, if we apply the adapted Carleman inequality (28) to $\rho_{\varepsilon}$, we obtain

$$
\int_{0}^{T} \int_{\Gamma} \frac{1}{\theta^{2}}\left|\frac{\partial \rho_{\varepsilon}}{\partial \nu}\right|^{2} d \Gamma d t \leq C \int_{0}^{T} \int_{\gamma}\left|(I-P) \frac{\partial \rho_{\varepsilon}}{\partial \nu} \chi_{\gamma}\right|^{2} d \Gamma d t
$$

From (47), the hypothesis on $h_{0}$ and the choice of $w_{0} \in Y_{\theta}$, we see that

$$
\begin{aligned}
& \frac{1}{\varepsilon}\left|q_{\varepsilon}(0)\right|_{H^{-1}(\Omega)}^{2}+\int_{0}^{T} \int_{\gamma}\left|(I-P)\left(\frac{\partial \rho_{\varepsilon}}{\partial \nu} \chi_{\gamma}\right)\right|^{2} d \Gamma d t \\
& \quad \leq C\left(\int_{0}^{T} \int_{\gamma}\left|w_{0}\right|^{2} d \Gamma d t+\int_{0}^{T} \int_{\gamma} \theta^{2}\left|w_{0}\right|^{2} d \Gamma d t+\int_{0}^{T} \int_{O} \theta^{2}\left|h_{0}\right|^{2} d \Gamma d t\right) .
\end{aligned}
$$

This implies

$$
\begin{aligned}
&\left|(I-P)\left(\frac{\partial \rho_{\varepsilon}}{\partial \nu} \chi_{\gamma}\right)\right| L^{2}(\gamma \times(0, T)) \\
& \leq C\left(\int_{0}^{T} \int_{\gamma} \theta^{2}\left|w_{0}\right|^{2} d \Gamma d t+\int_{0}^{T} \int_{O} \theta^{2}\left|h_{0}\right|^{2} d \Gamma d t\right)^{1 / 2}, \\
&\left|v_{\varepsilon}\right|_{L^{2}(\gamma \times(0, T))} \leq C\left(\int_{0}^{T} \int_{\gamma} \theta^{2}\left|w_{0}\right|^{2} d \Gamma d t+\int_{0}^{T} \int_{O} \theta^{2}\left|h_{0}\right|^{2} d \Gamma d t\right)^{1 / 2}, \\
&\left|q_{\varepsilon}(0)\right|_{H^{-1}(\Omega)} \leq C \sqrt{\varepsilon}\left(\int_{0}^{T} \int_{\gamma} \theta^{2}\left|w_{0}\right|^{2} d \Gamma d t+\int_{0}^{T} \int_{O} \theta^{2}\left|h_{0}\right|^{2} d \Gamma d t\right)^{1 / 2},
\end{aligned}
$$

where $C>0$ is independent of $\varepsilon$. Then, the properties of the heat equation (44) allow us to conclude that

$$
\left|q_{\varepsilon}\right|_{L^{2}(\mathcal{Q})} \leq C\left(\int_{0}^{T} \int_{\gamma} \theta^{2}\left|w_{0}\right|^{2} d \Gamma d t+\int_{0}^{T} \int_{O} \theta^{2}\left|h_{0}\right|^{2} d \Gamma d t\right)^{1 / 2} .
$$

From (49a) and (48), we see that

$$
\left|\frac{1}{\theta} \frac{\partial \rho_{\varepsilon}}{\partial \nu}\right|_{L^{2}(\Sigma)} \leq C\left(\int_{0}^{T} \int_{\gamma} \theta^{2}\left|w_{0}\right|^{2} d \Gamma d t+\int_{0}^{T} \int_{O} \theta^{2}\left|h_{0}\right|^{2} d \Gamma d t\right)^{1 / 2}
$$

and using again (49a) and the fact that $\frac{1}{\theta}$ is bounded, we obtain

$$
\left|\frac{1}{\theta} P \frac{\partial \rho_{\varepsilon}}{\partial \nu}\right|_{L^{2}(\gamma \times(0, T))} \leq C\left(\int_{0}^{T} \int_{\gamma} \theta^{2}\left|w_{0}\right|^{2} d \Gamma d t+\int_{0}^{T} \int_{O} \theta^{2}\left|h_{0}\right|^{2} d \Gamma d t\right)^{1 / 2}
$$

Therefore, $Y$ being a finite dimensional vector subspace of $L^{2}(\gamma \times(0, T))$, we deduce that

$$
\left|P \frac{\partial \rho_{\varepsilon}}{\partial \nu}\right|_{L^{2}(\gamma \times(0, T))} \leq C\left(\int_{0}^{T} \int_{\gamma} \theta^{2}\left|w_{0}\right|^{2} d \Gamma d t+\int_{0}^{T} \int_{O} \theta^{2}\left|h_{0}\right|^{2} d \Gamma d t\right)^{1 / 2} .
$$


Therefore, from (49a) we obtain

$$
\left|\frac{\partial \rho_{\varepsilon}}{\partial \nu}\right|_{L^{2}(\gamma \times(0, T))} \leq C\left(\int_{0}^{T} \int_{\gamma} \theta^{2}\left|w_{0}\right|^{2} d \Gamma d t+\int_{0}^{T} \int_{O} \theta^{2}\left|h_{0}\right|^{2} d \Gamma d t\right)^{1 / 2} .
$$

Using Proposition 2.3 we then have

$$
\begin{aligned}
\int_{0}^{T} \int_{\Omega} \frac{1}{\theta^{2}}\left(\left|\frac{\partial \rho_{\varepsilon}}{\partial t}\right|^{2}+\left|\Delta \rho_{\varepsilon}\right|^{2}\right. & \left.+\left|\nabla \rho_{\varepsilon}\right|^{2}+\left|\rho_{\varepsilon}\right|^{2}\right) d x d t \\
\leq & \leq\left(\int_{0}^{T} \int_{\gamma} \theta^{2}\left|w_{0}\right|^{2} d \Gamma d t+\int_{0}^{T} \int_{O} \theta^{2}\left|h_{0}\right|^{2} d \Gamma d t\right)
\end{aligned}
$$

- Step 3. In view of (49), (50), and (52), we can extract subsequences of $\left(v_{\varepsilon}\right),\left(q_{\varepsilon}\right)$, and $\rho_{\varepsilon}$ (still called $\left(v_{\varepsilon}\right),\left(q_{\varepsilon}\right)$, and $\left.\rho_{\varepsilon}\right)$ such that

$$
\begin{array}{lll}
v_{\varepsilon} \longrightarrow \tilde{v} & \text { weakly in } & L^{2}(\gamma \times(0, T)), \\
q_{\varepsilon} \longrightarrow \tilde{q} & \text { weakly in } & L^{2}(\mathcal{Q}), \\
\rho_{\varepsilon} \longrightarrow \tilde{\rho} & \text { weakly in } & L^{2}\left(\frac{1}{\theta}, \mathcal{Q}\right) .
\end{array}
$$

Therefore, $\tilde{v} \in Y^{\perp}, L^{*} q_{\varepsilon} \rightarrow L^{*} \tilde{q}$ weakly in $D^{\prime}(\mathcal{Q})$ and, in view of the first equality in (44), we have

$$
L^{*} \tilde{q}=0 \quad \text { in } \mathcal{Q} .
$$

Thus, $\tilde{q} \in L^{2}(\mathcal{Q})$ and $L^{*} \tilde{q} \in L^{2}(\mathcal{Q})$. Consequently, we can give a sense to the traces $\tilde{q}$ on $\Sigma$ and $\frac{\partial \tilde{q}}{\partial \nu}$ on $\Sigma$ respectively in $H^{-1}\left(0, T, H^{-1 / 2}(\Gamma)\right)$ and in $H^{-1}\left(0, T, H^{-3 / 2}(\Gamma)\right)$ on the one hand, and to $\tilde{q}(T)$ and $\tilde{q}(0)$ in $H^{-1}(\Omega)$ on the other hand. Now, it can be shown that

$$
q_{\varepsilon}(0) \longrightarrow \tilde{q}(0) \quad \text { in } \mathcal{D}^{\prime}(\Omega)
$$

but from (49c) we know that

$$
q_{\varepsilon}(0) \longrightarrow 0 \text { in } H^{-1}(\Omega) \text { strongly }
$$

so that

$$
\tilde{q}(0)=0 .
$$

Therefore it is clear that $(\tilde{v}, \tilde{q})$ verifies $(14)-(16)$ and there exists a solution to the boundary null-controllability problem. Moreover, it is clear from (45) that $\tilde{\rho}$ satisfies

$$
\left\{\begin{array}{lll}
L \tilde{\rho}=0 & \text { in } \quad \mathcal{Q} \\
\tilde{\rho}=0 & \text { on } \quad \Sigma
\end{array}\right.
$$


Notice that $\tilde{\rho}$ may not have an initial value at time 0 . Nevertheless because of (51) we see that

$$
\frac{\partial \rho_{\varepsilon}}{\partial \nu} \longrightarrow \frac{\partial \tilde{\rho}}{\partial \nu} \quad \text { weakly in } \quad L^{2}(\gamma \times(0, T))
$$

and therefore

$$
\tilde{v}=(I-P)\left(w_{0} \chi_{\gamma}-\frac{\partial \tilde{\rho}}{\partial \nu} \chi_{\gamma}\right) .
$$

As there exists a solution to the boundary null-controllability problem, it has already been noticed that we can find a unique $\hat{v} \in \mathcal{E}$ (admissible control) such that $\left(w_{0}-\hat{v}\right)$ is of minimal norm in $L^{2}(\gamma \times(0, T))$. If we denote by $\hat{q}$ the corresponding solution of (15), we have $\hat{q}(0)=0$ and, as $\tilde{v} \in \mathcal{E}$,

and

$$
\frac{1}{2}\left|w_{0}-v_{\varepsilon}\right|_{L^{2}(\gamma \times(0, T))}^{2} \leq J_{\varepsilon}\left(v_{\varepsilon}\right) \leq J_{\varepsilon}(\hat{v})=\frac{1}{2}\left|w_{0}-\hat{v}\right|_{L^{2}(\gamma \times(0, T))}^{2}
$$

$$
\frac{1}{2}\left|w_{0}-\hat{v}\right|_{L^{2}(\gamma \times(0, T))}^{2} \leq \frac{1}{2}\left|w_{0}-\tilde{v}\right|_{L^{2}(\gamma \times(0, T))}^{2} .
$$

But because of (53),

$$
\liminf _{\varepsilon \rightarrow 0} \frac{1}{2}\left|w_{0}-v_{\varepsilon}\right|_{L^{2}(\gamma \times(0, T))}^{2} \geq \frac{1}{2}\left|w_{0}-\tilde{v}\right|_{L^{2}(\gamma \times(0, T))}^{2} .
$$

Therefore we have

$$
\tilde{v}=\hat{v}
$$

and

$$
v_{\varepsilon} \longrightarrow \hat{v} \quad \text { strongly in } \quad L^{2}(\gamma \times(0, T)) .
$$

Writing $\hat{\rho}=\tilde{\rho}$ we have

$$
\hat{v}=(I-P)\left(w_{0} \chi_{\gamma}-\frac{\partial \hat{\rho}}{\partial \nu} \chi_{\gamma}\right) .
$$

This finishes the proof of Theorem B.

\section{References}

[1] B.-E. Ainseba, J.-P. Kernévez, and R. Luce, Identification de paramètres dans les problèmes non linéaires à données incomplètes, RAIRO Modél. Math. Anal. Numér. 28 (1994), no. 3, 313-328 (French, with English and French summaries).

[2] O. Bodart and P. Demeestere, Sentinels for the identification of an unknown boundary, Math. Models Methods Appl. Sci. 7 (1997), no. 6, 871-885.

[3] T. Cazenave and A. Haraux, Introduction aux problèmes d'évolution semi-linéaires, Mathématiques \& Applications, vol. 1, Ellipses, Paris, 1990.

[4] E. Fernández-Cara and S. Guerrero, Global Carleman inequalities for parabolic systems and applications to controllability, SIAM J. Control Optim. 45 (2006), no. 4, 1399-1446. 
[5] A. V. Fursikov and O. Yu. Imanuvilov, Controllability of evolution equations, Lecture Notes, vol. 34, Research Institute of Mathematics, Seoul National University, Seoul, 1996.

[6] O. Yu. Imanuilov, Controllability of parabolic equations, Mat. Sb. 186 (1995), no. 6, 109-132 (Russian); English transl., Sb. Math. 186 (1995), no. 6, 879-900.

[7] J.-P. Kernévez, The sentinel method and its application to environmental pollution problems, CRC Mathematical Modelling Series, CRC Press, Boca Raton, FL, 1997.

[8] G. Lebeau and L. Robbiano, Contrôle exact de l'équation de la chaleur, Comm. Partial Differential Equations 20 (1995), no. 1-2, 335-356.

[9] Y.-J. Lin Guo and W. Littman, Null boundary controllability for semilinear heat equations, Appl. Math. Optim. 32 (1995), no. 3, 281-316.

[10] J.-L. Lions, Sentinelles pour les systèmes distribués à données incomplètes, Recherches en Mathématiques Appliquées, vol. 21, Masson, Paris, 1992.

[11] _ Optimal control of systems governed by partial differential equations, Grundlehren der mathematischen Wissenschaften, vol. 170, Springer-Verlag, New York, 1971.

[12] J.-L. Lions and E. Magenes, Problèmes aux limites non homogènes et applications. 1, Travaux et Recherches Mathématiques 17; 2, Travaux et Recherches Mathématiques 18, Dunod, Paris, 1968.

[13] S. Mizohata, Unicité du prolongement des solutions pour quelques opérateurs différentiels paraboliques, Mem. Coll. Sci. Univ. Kyoto. Ser. A. Math. 31 (1958), 219-239.

[14] O. Nakoulima, Contrôlabilité à zéro avec contraintes sur le contrôle, C. R. Math. Acad. Sci. Paris 339 (2004), no. 6, 405-410 (French, with English and French summaries).

[15] D. L. Russell, A unified boundary controllability theory for hyperbolic and parabolic partial differential equations, Studies in Appl. Math. 52 (1973), 189-211.

[16] J.-C. Saut and B. Scheurer, Unique continuation for some evolution equations, J. Differential Equations 66 (1987), no. 1, 118-139. 